\title{
The impact of alphabet size on pattern complexity of maxmin- $\omega$ cellular automata
}

\author{
Ebrahim L. Patel ${ }^{1}$ \\ Published online: 14 April 2020 \\ (C) The Author(s) 2020
}

\begin{abstract}
We present an analysis of an additive cellular automaton (CA) under asynchronous dynamics. The asynchronous scheme is maxmin- $\omega$, a deterministic system, introduced in our previous work with a binary alphabet. Extending this work, we study the impact of a larger alphabet, which also allows a meaningful inference of the behaviour of the resultant CA from the asymptotic behaviour of the maxmin- $\omega$ update system. Far from being a straightforward positive correlation between complexity and alphabet size, we show that there is a region of $\omega$ and alphabet size where complexity of CA is maximal. Thus, despite employing a fixed CA rule, the complexity of this CA can be controlled by $\omega$ and alphabet size. The main message is that the effect of maxmin- $\omega$ updating on the state of a network can be well understood, especially if the state alphabet is counter-intuitively large.
\end{abstract}

Keywords Max-plus algebra $\cdot$ Cellular automaton · Alphabet · Entropy $\cdot$ Complexity

\section{Introduction}

The maxmin- $\omega$ system was introduced in Patel (2016) as a model of asynchronous dynamics on networks. Each node in this system updates its state upon receiving input from a proportion $\omega$ of neighbourhood nodes. In terms of a cellular automaton (CA) application, the main attraction for this type of asynchrony is its determinism; here, 'receiving neighbourhood inputs' corresponds to knowing the CA states of a cell's neighbours after some delay. Maxmin- $\omega$ is therefore not only a departure from traditional asynchronous CA schemes (e.g., Schönfisch and de Roos (1999) and Fatès and Morvan (2005)) but differs from recent work that similarly considers the receipt of such neighbourhood information, but where this receipt is stochastic (Bouré et al. 2012). Moreover, by thinking of $\omega$ as a threshold that must be met before this neighbourhood information is processed, maxmin- $\omega$ provides a simpler and intuitive mechanism for asynchrony, mimicking the dynamics of similar models whose applications include

Ebrahim L. Patel

ebrahim.patel@maths.ox.ac.uk

1 Mathematical Institute, University of Oxford, Oxford OX2 6GG, UK neuronal networks (McCulloch and Pitts 1943; Wilson and Cowan 1972; Thul et al. 2016) and virus transmission (Granovetter 1978; Watts 2002; Backlund et al. 2014). Taking these points together, maxmin- $\omega$ looks like a new member of the class of threshold models that have their roots in epidemic spreading (Granovetter 1978).

We briefly describe the system here. Consider a onedimensional CA lattice. This lattice has a natural definition of neighbourhood, i.e., the neighbourhood $\mathcal{N}_{i}$ of cell $i$ of radius $r$ is $\{i-r, \ldots, i-1, i, i+1, \ldots, i+r\}$, as introduced in Wolfram (1983). Maxmin- $\omega$ views the CA lattice as a network, whose nodes play the role of cells. Thus, a cell state is updated at the end of a cycle. The processes that constitute such a cycle are as follows. First, the neighbourhood nodes $\mathcal{N}_{i}$ complete their $k$ th cycle, $k \in\{0,1,2, \ldots\}$, and then transmit their CA state to node $i$; the transmission of such a state from node $j$ to $i$ takes transmission time $\tau_{i j}$. (Note that $k=0$ corresponds to the initialisation of the system, where all nodes simply transmit their initial states.) Node $i$ waits for a fraction $\omega$ of the arriving states before processing its new CA state, which takes processing time $\xi_{i}$. Once this is complete, the node updates its CA state and simultaneously transmits this state to downstream nodes, where the cycles are reiterated. Fig. 1 graphically shows this state change process. Thus, 


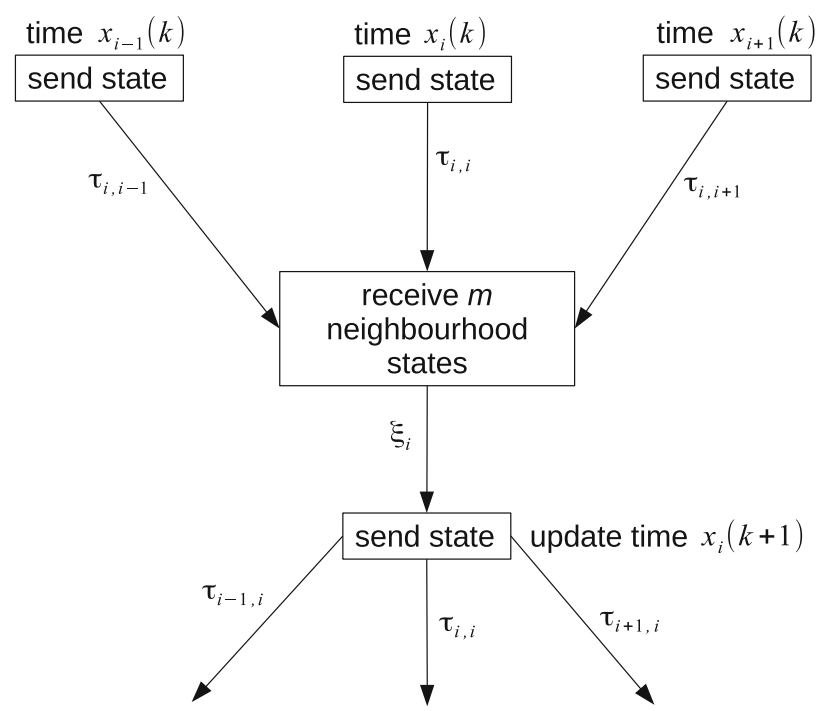

Fig. 1 The processes within the $k$ th cycle that yield a state change for cell $i$ due to maxmin- $\omega$. Assuming a neighbourhood size $n$, we take $m=\lceil\omega n\rceil$

the delay parameters $\tau_{i j}$ and $\xi_{i}$ are central to the asynchronous receipt of neighbourhood states; the fastest arriving states are processed.

For an ordered set of $n$ objects and $\omega \in(0,1]$, let us define the $\omega$ th object as the $m$ th object where $m=\lceil\omega n\rceil$. (For example, the 0.25th object in the set $\{3,3.2,5,9,15.8\}$ is 3.2.) We denote the time of state change of node $i$ by $x_{i}(k+1)$, whilst the CA state of node $i$ in the same cycle is denoted $s_{i}(k+1)$. Given the temporally ordered set of arrival times of all neighbourhood inputs in cycle $k$, the $(k+1)$ th update time of node $i$ is given by the following recurrence relation.

$x_{i}(k+1)=x_{(\omega)}(k)+\xi_{i}$

where $x_{(\omega)}(k)$ represents the $k$ th time of arrival of the $\omega$ th input from the neighbourhood of $i$; if $k$ is clear from context, we denote this $x_{(\omega)}$ for short.

For our study, we employ an additive CA rule as follows. We first consider an alphabet of CA states taking size $Z$, namely $\Sigma=\{0,1,2, \ldots, Z-1\}$. We represent the (CA) state of the system at cycle $k \in \mathbb{N}$ by the vector $\mathbf{s}(k)=\left(s_{1}(k), s_{2}(k), \ldots, s_{N}(k)\right)$. Our CA rule is a function $f:\{0,1\}^{2 r+1} \rightarrow\{0,1\}$ given by $s_{i}(k+1)=f\left(\mathcal{N}\left(s_{i}(k)\right)\right)$, where $\mathcal{N}\left(s_{i}(k)\right)$ denotes the CA states of $\mathcal{N}_{i}$ in cycle $k$. Further, consider

$\mathcal{A}_{i}(k)=\left\{j \in \mathcal{N}_{i}: x_{j}(k)+\tau_{i j} \leq x_{(\omega)}(k)\right\}$

which is the set of all nodes whose CA states arrive before or at the same time as the $\omega$ th input at node $i$. We call $\mathcal{A}_{i}(k)$ the set of affecting nodes of $i$. We focus on the following $\mathrm{CA}$ rule $s_{i}(k+1)=\sum_{j \in \mathcal{A}_{i}} s_{j}(k) \bmod Z$.

Simply put, the CA state of each cell will be the sum of the fastest arriving states at that cell. The main reason for using rule (3) is that it is simple to understand and analyse. Under certain conditions, however, such additive CA have been known to exhibit complex behaviour (Manzini and Margara 1999; Chaudhuri et al. 1997; Aso and Honda 1985; Cattaneo et al. 2000; Guan and He 1986). Importantly for this paper, due to their simplicity, such CA can be used to investigate chaos (Cattaneo et al. 2004; Dennunzio et al. 2019). In a similar vein, we want to investigate the sensitivity of CA to the parameter $\omega$; an additive CA gives a transparent and uncomplicated rule to enable this study.

We make special note that $k$ is a discrete quantity, with the state of a cell remaining constant between successive cycles $k$ and $k+1$. Thus, there is an underlying real time $t \in \mathbb{R}$, which is punctuated by cycles $k$. This means that there are two ways to index the time-by its real time value or by the counter value $k$; in this way, $k$ may also be referred to as an epoch. Overall, we can denote the state of a cell $i$ in two ways: $s_{i}(k)$ is the state on epoch $k$ whilst $s_{i}^{(t)}$ is the state at real time $t$. Thus, for example, on epoch $k=2$, if $t=5.8$, we have $s_{i}(2)=s_{i}^{(5.8)}$. These epochs are depicted as contours in Fig. 2. If the CA updating was synchronous, these contours would be horizontal, and all cells would process the same epoch at the same time; under maxmin- $\omega$ asynchrony, at some real time $t$, two cells can be in different epochs. Notice that, even though the same number of iterations are taken of (1), the system runs for a longer real time for larger $\omega$; this is as expected since larger $\omega$ implies waiting for more inputs before updating.

We exhibited the impact of maxmin- $\omega$ on CA with a binary alphabet in Patel (2016), i.e., $\Sigma=\{0,1\}$. Figure 2 shows an example of such a binary CA. In this paper, we demonstrate the difference in effect for an extended alphabet, i.e., $|\Sigma|>2$. In particular, we ask the question: what is the effect of maxmin- $\omega$ on the complexity of cellular automata?

In Sect. 2, we present a measure of CA space-time pattern complexity, and make analytical predictions for the impact of the alphabet size on complexity. Section 3 introduces a reduced form of the maxmin- $\omega$ system that allows us to make heuristic predictions of the impact of $\omega$ on CA complexity. Section 4 presents the results from simulations of maxmin- $\omega \mathrm{CA}$; these results are compared with the theory of the previous two sections. We discuss the work in Sect. 5, giving arguments for the results obtained and potential avenues for future work that maxmin- $\omega$ opens up. 

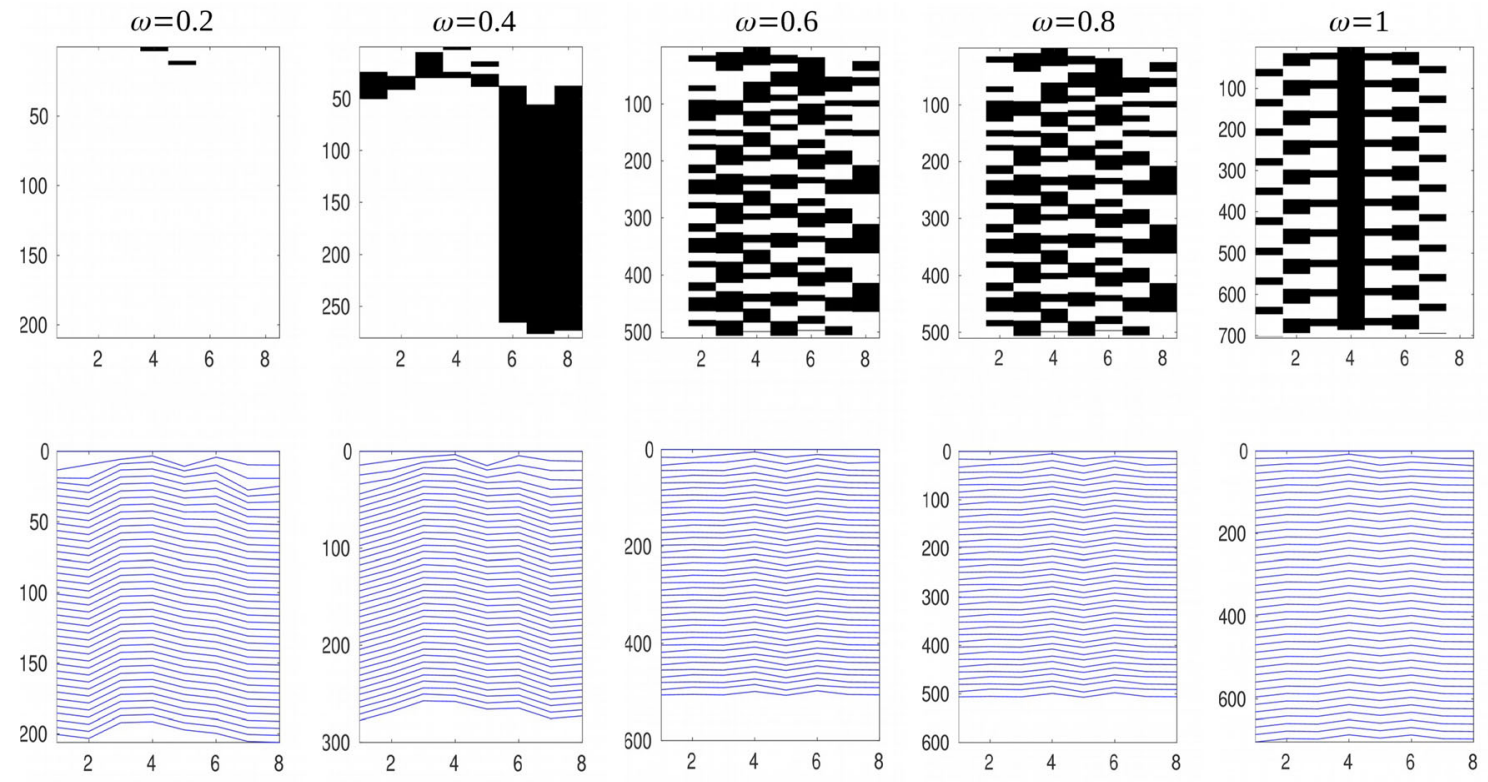

Fig. 2 Above: Binary CA space-time patterns as a function of $\omega$; state 0 is coloured white, state 1 is black. Below: Update times, depicted as contours, of the CA above. Contour $k$ connects the update times $x_{i}(k)$ and $x_{i+1}(k)(y$-axis) of nodes $i$ and $i+1(i=1, \ldots, 9)$. In all cases, the

\section{Cellular automata with general alphabet}

In the analysis of cellular automata, the fundamental breakthroughs have been demonstrated with binary states, i.e., $\Sigma=\{0,1\}$. Whilst this continues to provide informative results, it is instructive to expand the state possibilities. Not only would this allow an analysis of the generalised system-with hypotheses for binary $\Sigma$ being tested for general $\Sigma$-but, importantly, potential applications would be better reflected. Examples include models of traffic flow, where cars (represented by cells) can take on a range of velocities (Nagel and Schreckenberg 1992); epidemic models often assume a person to be in one of three states susceptible, infected, or recovered (White et al. 2007); and cancerous tumour growth models take cells to be in one of the proliferating, quiescent and necrotic states, and possibly more, depending on the accuracy desired (Piotrowska and Angus 2009; Gevertz and Torquato 2006).

\subsection{Cellular automaton pattern complexity}

To classify our CA in space-time, we use the entropy measures of Marr and Hütt (2005). The Shannon entropy $S$ relies on the density $p\left(s_{j}\right)$ of the CA state $s_{j} \in \Sigma$ in the time series of the evolving CA states of each cell. Thus, for example, given $|\Sigma|=\{0,1,2,3\}$ and the following time series of length 15 , lattice has size 8, with $r=2$; we take 40 iterations of (1) and (3) (so there are 41 contours displayed for each $\omega)$ with $\mathbf{x}(0)=(0, \ldots, 0)$ and $s_{4}(0)=1, s_{i}(0)=0$ for $i \neq 4$; the $x$-axis denotes the node number, and (real) time travels down

we have $p(0)=3 / 15, p(1)=2 / 15, p(2)=4 / 15$, and $p(3)=6 / 15$. The Shannon entropy of cell $i$ is defined as

$S_{i}=-\sum_{j=1}^{|\Sigma|} p\left(s_{j}\right) \log _{2} p\left(s_{j}\right)$.

For the example time series (4), $S_{i}=1.8892$. The quantity we require is the Shannon entropy of the overall CA spacetime pattern, defined as the average of $S_{i}$ over the $N$ cells in the lattice: $S=\frac{1}{N} \sum_{i=1}^{N} S_{i}$.

The word entropy $W$ depends on the occurrence of blocks of constant states of length $l$ ( $l$-words) in the time series of a cell. The $l$-words we consider are independent of the state comprising them, e.g., in the example (4), there are eight 1 -words (namely 1, 2, 1, 0, 0, 2, 3, 0, in that order), two 2 -words (22 and 33), one 3 -word (333), and no words of longer length. Thus, if $p(l)$ is the density of an $l$ word along the time series of a cell $i$, then

$$
W_{i}=-\sum_{l=1}^{T} p(l) \log _{2} p(l)
$$

where $T$ is the length of the time series. Using (4) again to illustrate, there are eleven words in total such that $p(1)=8 / 11, p(2)=2 / 11$, and $p(3)=1 / 11$. The word entropy of the entire CA pattern is then defined as the average of $W_{i}$ over the $N$ cells: $W=\frac{1}{N} \sum_{i=1}^{N} W_{i}$. 
We make a crucial note that the entropy measures used here should not be interpreted in the standard informationtheoretical or thermodynamical way. This is mainly due to the finiteness in the time series that we analyse, the averaging over individual entropies, and the strict definition of $W_{i}$ such that it is not bounded to $[0,1]$.

Marr and Hütt themselves acknowledge such drawbacks in Marr and Hütt (2005) and Marr et al. (2007). However, they go on to advocate their $S$ and $W$ measures since they are generically applicable to arbitrary topologies (Marr and Hütt 2005) and "their combination was found to perform well in separating different dynamic regimes" (Marr et al. 2007). It is this amenability to generalisations that attracts us to the employment of these entropy measures. We envisage the latter point to be particularly pronounced as $|\Sigma|$ increases in this paper, whilst the first point of applications to different networks forms the basis of current research. Of course, there are other classification schemes, such as those that deal with specific types of CA output Dennunzio et al. (2013a), Cattaneo et al. (2002), and those that approach it from the angle of computational complexity (for example, Dennunzio et al. 2017). Notwithstanding this, the entropic measure of complexity employed in this paper can be best compared with that of LMC complexity (Lopez-Ruiz et al. 1995) and Rènyi entropy (Rényi 1961) (see later in this section).

Additive rules have the same Langton parameter $\lambda_{s}$ for all states $s \in \Sigma$ (Gutowitz and Langton 1995). In other words, in the look-up table for the additive CA rule (3) that we consider, each state is equally likely (the look-up table lists all possible neighbourhood states and their transitions according to (3)). Suppose this equiprobability is true also in the time series evolution of such a CA. Then we have that $p\left(s_{j}\right)=1 / Z$, giving

$S_{i}=-\sum_{j=1}^{Z} \frac{1}{Z} \log \left(\frac{1}{Z}\right)$

$=\log Z$.

Taking the average of this quantity over $N$ cells gives $S=\log Z$. Although it is not as straightforward to calculate the exact densities of states in a time series, the assumption of equiprobability of states gives a Shannon entropy that is maximal, as is the case for a thermodynamic entropy, that is,

Proposition 1 The Shannon entropy is maximal when all CA states are equiprobable (see "Appendix" for proof).

Therefore, (8) tells us that, in the extremal case, $S$ is expected to increase logarithmically with the size of alphabet.
As for the word entropy $W$, it is reliant on the state in a time series of a cell being unchanged over some fixed length $l$ of time. Recall $s_{j}^{(t)}$, the state of cell $j$ at time $t \in \mathbb{R}$. Thus, an $l$-word satisfies the following.

$s_{j}^{(t)}=s_{j}^{(t+1)}=\cdots=s_{j}^{(t+l-1)}$

where $s_{j}^{(t-1)} \neq s_{j}^{(t)}$ and $s_{j}^{(t)} \neq s_{j}^{(t+l)}$. Note that, despite defining $t$ as a continuous real index of time, here we have considered the state of cell $i$ at discrete points of time with step size 1; this will be sufficient to demonstrate our theory. Assuming the extremal $S$ case again, where all CA states are equally likely, $p\left(s_{j}^{(t)}\right)=1 / Z$ for all $t$. The probability of the next state being the same is $P\left(s_{j}^{(t)}=s_{j}^{(t+1)}\right)=1 / Z$, whilst the probability of the next state being different is $P\left(s_{j}^{(t)} \neq s_{j}^{(t+1)}\right)=\frac{Z-1}{Z}$. Then the probability $p(l)$ of observing an $l$-word is $P\left(s_{j}^{(t-1)} \neq s_{j}^{(t)}=s_{j}^{(t+1)}=\cdots=\right.$ $\left.s_{j}^{(t+l-1)} \neq s_{j}^{(t+l)}\right)$, calculated as

$$
\frac{Z-1}{Z} \cdot \underbrace{\frac{1}{Z} \cdot \cdots \cdot \frac{1}{Z}}_{l \text { times }} \cdot \frac{Z-1}{Z}=\frac{(Z-1)^{2}}{Z^{l+2}} \text {. }
$$

Using earlier notation, we have $p(l)=\frac{(Z-1)^{2}}{Z^{l+2}}$. The inset of Fig. 3 plots $p(l)$ as a function of $Z$ and $l$ for various values of $l$ and $Z$ respectively. Figure 4 shows histograms of word lengths arising from the CA time series of one cell in four example systems (each taking different alphabet sizes). The correspondence with the left hand inset of Fig. 3 is clear; 1 -words in particular are most frequent.

Substituting $p(l)$ into (6) gives $W_{i}$, the word entropy of each cell; taking the average over all cells gives $W=W_{i}$ here. Figure 3 plots $W$ as a function of $|\Sigma|$. The word entropy is, thus, expected to decrease as the alphabet size increases.

For comparison, let us briefly discuss the LMC complexity $C$ from Lopez-Ruiz et al. (1995). Since we consider two measures characterised by individual cell states and blocks of cell states in a time series, we carry this concept over to construct two parallel LMS measures: (i) $C_{S}=S D_{S}$, where $D_{S}=\Sigma_{j=1}^{Z}\left(p\left(s_{j}\right)-1 / Z\right)^{2}$ is a measure of 'disequilibrium', and (ii) $C_{W}=W D_{W}$, where $D_{W}$ is a measure of disequilibrium, given by $D_{W}=\Sigma_{l=1}^{T}(p(l)-1 / T)^{2}$. The values $1 / Z$ and $1 / T$ respectively denote the probability of observing a CA state and an $l$-word if all states and all words are equiprobable. Since our additive rules imply $p\left(s_{j}\right)=1 / Z$ for all $j$, we have $D_{S}=0$ so that $C_{S}=0$. To calculate $C_{W}$ we substitute $p(l)=\frac{(Z-1)^{2}}{Z^{l+2}}$ in $D_{W}$ to obtain a decreasing $C_{W}$ with alphabet size. Thus, in the language of Lopez-Ruiz et al. 
Fig. 3 Word entropy (6) as a function of alphabet size $Z$, for $Z \leq 10$. The underlying $C A$ system is iterated 100 times, so that the largest word length possible is 100 and $T=100$ in (6). Inset, left: $p(l)$ against word length $l$ for alphabet sizes 2 to 5 . Inset, right: $p(l)$ as a function of alphabet size for $l=1, \ldots, 8$, where a small $l$ gives a higher curve

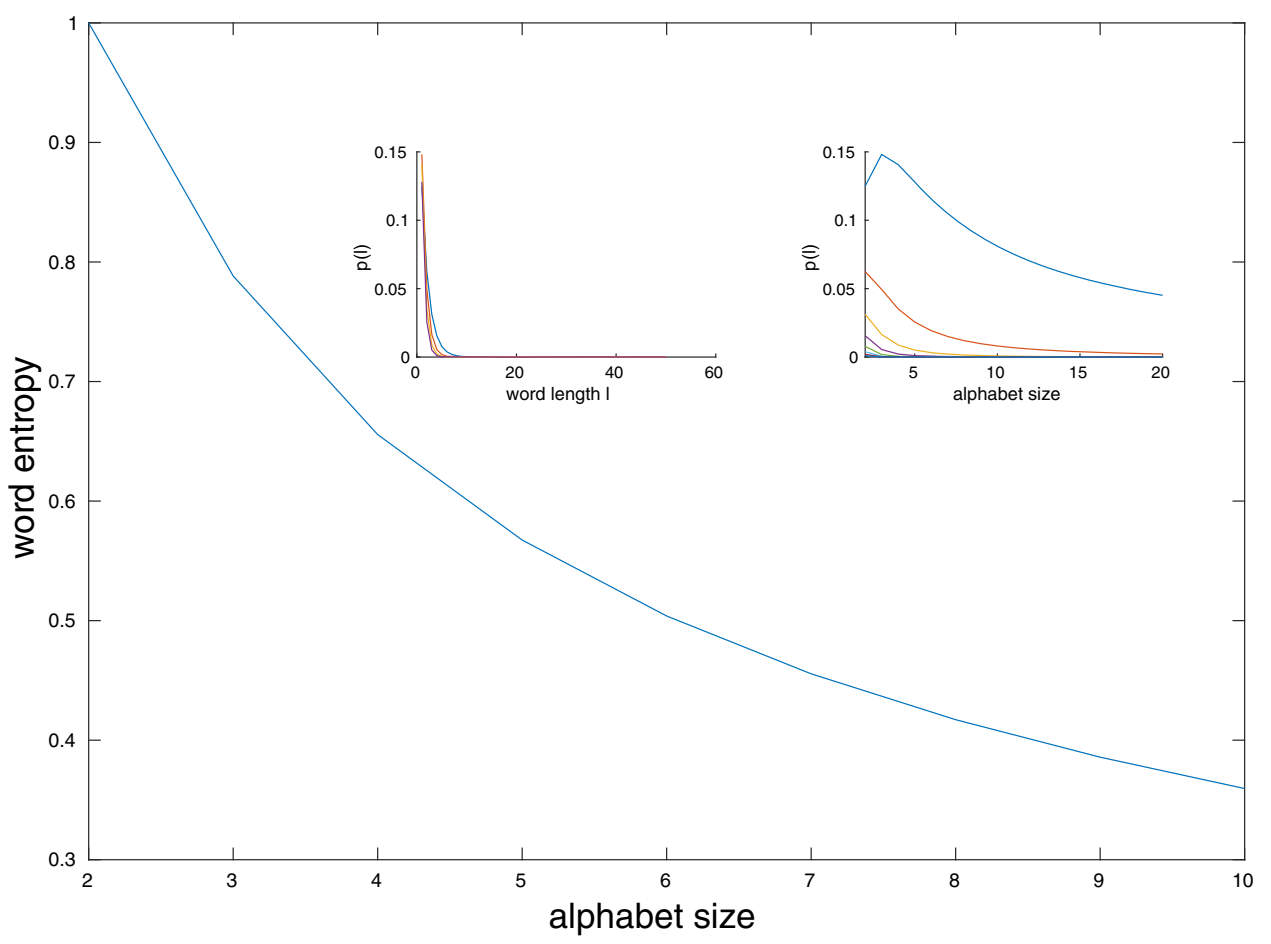

(1995), both $C_{S}$ and $C_{W}$ tend to behave like ideal gases, where a variety of CA patterns are observed.

The LMC complexity as a function of $\omega$ is more interesting. We predict that, for small $\omega$, since only a few cell states prevail (the fastest ones), then the overall behaviour is akin to a crystal-predictably periodic; as $\omega$ increases, more CA states become admissable, giving a wider probability distribution, though each non-zero probability is equal. Thus, $D_{S}$ is decreasing with $\omega$ and, since $S$ is increasing with $\omega$, we have an intriguing situation to that of Fig. 1 of Lopez-Ruiz et al. (1995), with $C_{S}$ maximising for some value of $\omega$ between 0 and 1 , and disappearing when $\omega=0$ and 1 . Since $p(l)$ is expected to decrease with $\omega$, we have $D_{W} \rightarrow 0$ as $\omega$ increases. Therefore, $C_{W} \rightarrow 0$ as $\omega \rightarrow 1$.

\section{Cellular automata as a function of $\omega$}

How do we expect the maxmin- $\omega$ system to affect CA output? We try to answer this question by looking at the impact of $\omega$ on the complexity of additive CA space-time output. The nature of complexity makes prediction a difficult science. We can, however, speculate on the tendency of CA behaviour as a function of $\omega$. Whilst we know about maxmin- $\omega$ on a binary CA (Patel 2016, 2012), here we focus on the effect of a larger alphabet on the Shannon and word entropies.

We first introduce the concept of a reduced network. Recall $\mathcal{A}_{i}(k)$, the set of affecting nodes of $i$ in cycle $k$.
Definition 3.1 In cycle $k$, the reduced network is the set of affecting nodes $\mathcal{A}_{i}(k)$ of all nodes, together with the edges that connect affecting nodes $j \in \mathcal{A}_{i}(k)$ to their affected node $i$.

In other words, the reduced network does not contain those nodes and edges whose states arrive too late, i.e., after the $\omega$ th input. We can draw up a reduced network for each counter $k$. In Patel (2012), we show that this sequence of reduced networks asymptotically settles onto a fixed set of reduced networks. Formally, let us denote by $\mathcal{G}^{r}(k)$ the reduced network in cycle $k$. Then we obtain the sequence $\mathcal{G}^{r}(0), \mathcal{G}^{r}(1), \mathcal{G}^{r}(2), \ldots$ of reduced networks such that, for some $k \geq 0$, there exists $g \in \mathbb{N}$ such that $\mathcal{G}^{r}(k+g)=\mathcal{G}^{r}(k)$. The set $\mathcal{O}=\left\{\mathcal{G}^{r}(k), \mathcal{G}^{r}(k+1), \cdots\right.$, $\left.\mathcal{G}^{r}(k+g)\right\}$ is called a periodic orbit of reduced networks. This set is dependent on the initial set of update times $\mathbf{x}(0)=\left(x_{1}(0), x_{2}(0), \ldots, x_{N}(0)\right)$ of the maxmin- $\omega$ system Patel (2012). Figure 5 shows an example of such a sequence of reduced networks that enter a periodic orbit of size two; here the original network is a size 3 fully connected regular network (with neighbourhood size 3 ), whilst the system takes $\omega=2 / 3$.

Pertinently, this means that, as $k \rightarrow \infty$, the maxmin- $\omega$ system can be replaced by a reduced system (with an underlying reduced network) with $\omega=1$. This is intuitive-since only the affecting nodes affect the future state of a node $i$, it is equivalent to node $i$ accepting all (such that $\omega=1)$ inputs from $\mathcal{A}_{i}(k)$. 
$|\Sigma|=2$
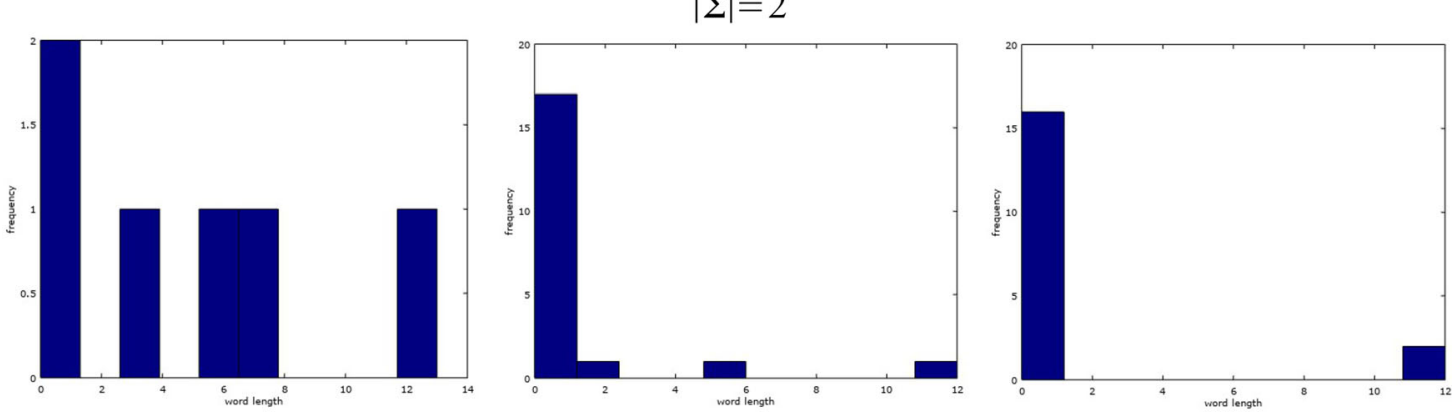

$|\Sigma|=5$
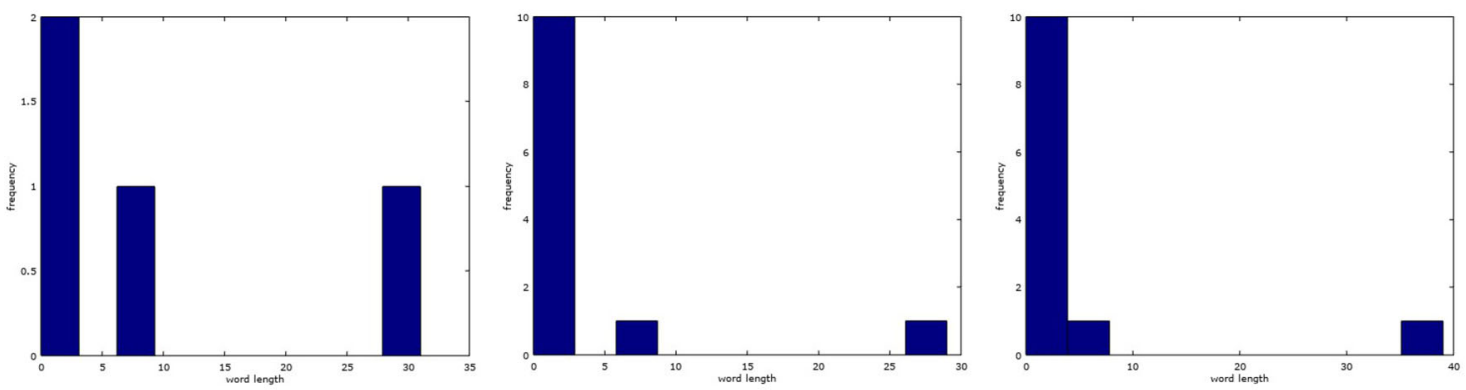

$|\Sigma|=10$
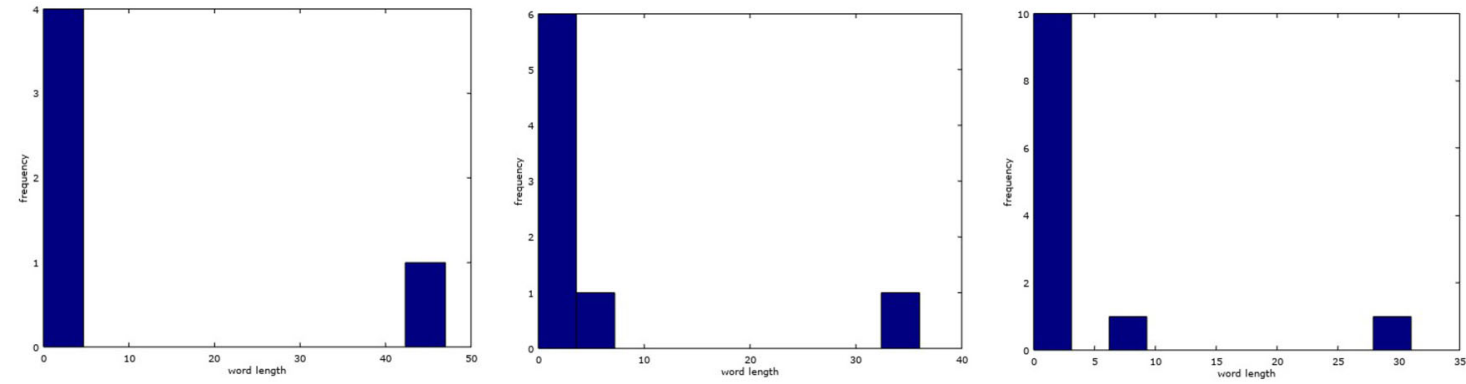

$|\Sigma|=20$
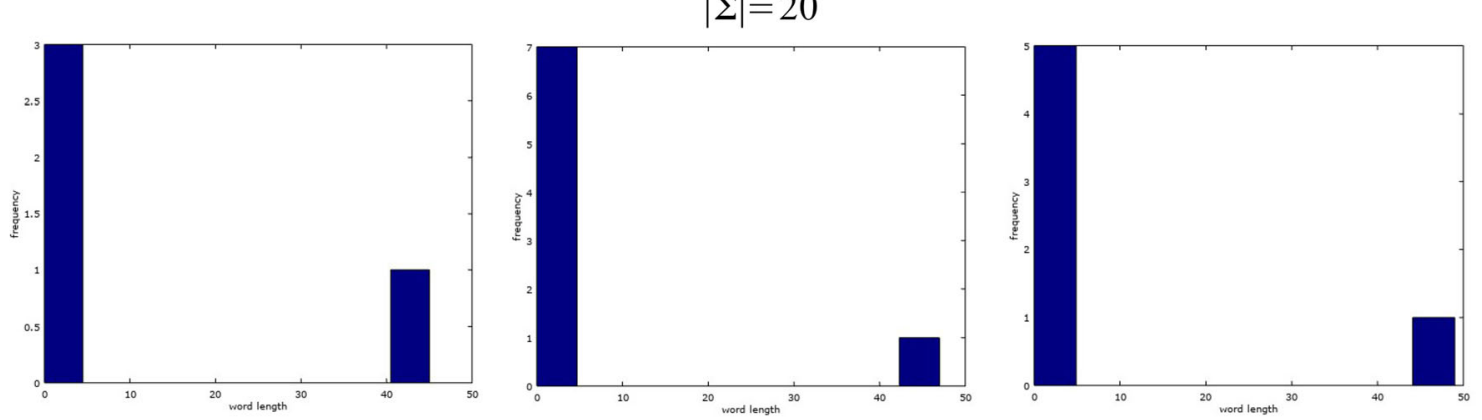

Fig. 4 Histograms giving the frequency of words obtained in the CA time series of the 20th cell after 50 iterations of the maxmin- $\omega$ system. The underlying lattice has size 20 and neighbourhood size $n=9$; transmission times are taken as random integers up to 5 , whilst processing times are all 1 . Histograms from left to right respectively correspond to $\omega=0.05,0.5$, and 1 . Alphabet sizes increase from top to bottom: $|\Sigma|=2,5,10$, and 20 , whilst initial CA states are taken uniformly at random from $\Sigma$ 


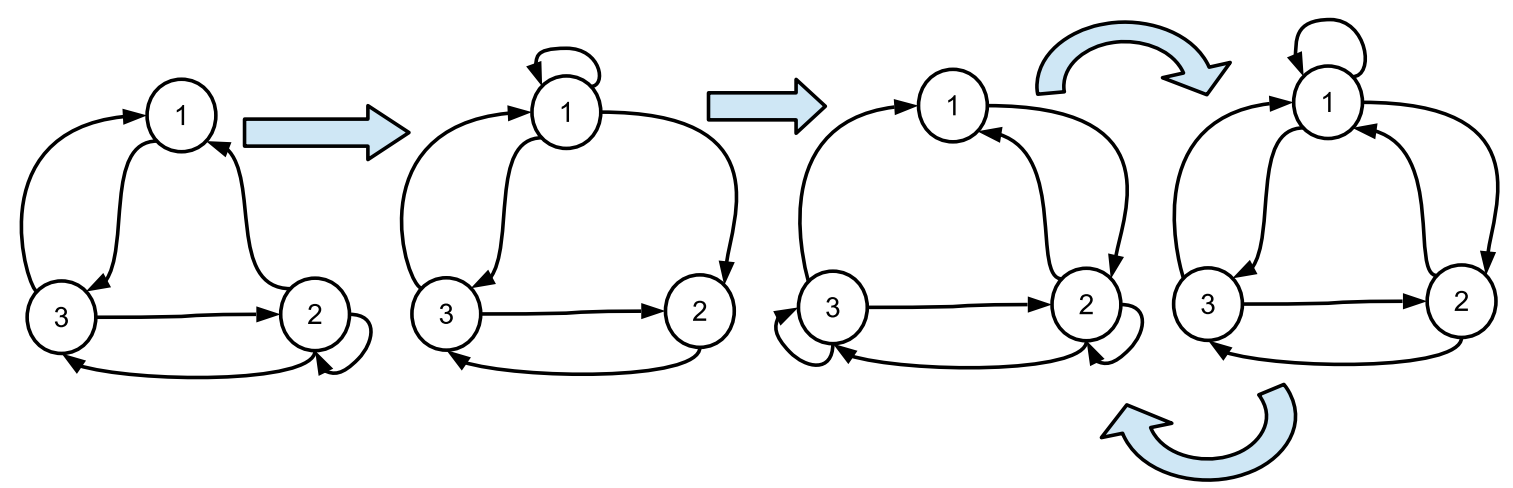

Fig. 5 Sequence of reduced networks of a maxmin-2/3 system where $N=3$. Larger arrows indicate the transitions between successive iterations of the maxmin- $2 / 3$ system

The implication on the asymptotic CA state of nodes can now be discussed. Once the idea of affecting nodes and the reduced network is understood, the size of each affecting node set can be inferred as $\left|\mathcal{A}_{i}(k)\right| \approx m$, where $m=\lceil\omega n\rceil$ (and recall that $n$ is the neighbourhood size of each node in the original network). This implies that the neighbourhood size of each node in $\mathcal{G}^{r}(k)$ should be approximately $m$. In fact, due to the simultaneous arrival of some affecting nodes, $\left|\mathcal{A}_{i}(k)\right| \geq m$ (Patel 2012). Nevertheless, it is instructive to assume the average neighbourhood size of each node in a reduced network to be $m$ (for example, see Fig. 5, where most nodes take neighbourhood size $m=2$ ). Instead of $\omega$, we can now think about the effect of $m$.

For further illustration, it is sufficient to take $g=1$, i.e., the periodic orbit of the asymptotic reduced network takes size 1 . Asymptotically then, we need only consider one underlying network; from here onwards, we shall take all mentions of "reduced network" to refer to the asymptotic reduced network, denoted $\mathcal{G}^{r}$. Thus, when $m$ is small, the neighbourhood size of each node in $\mathcal{G}^{r}$ is small. This implies that some CA states-namely the fastest arriving ones-are favoured over other states. Consequently, the cellular automaton state space is narrower, giving fewer state possibilities and therefore a smaller Shannon entropy. Assuming the extremal case again-that those CA states that appear with non-zero probability are equiprobablewe can use (8), which says that $S=\log Z$, where $Z$ is the number of $C A$ states. That is, the maximal Shannon entropy increases with the number of states. Thus, we obtain the following proposition.

Proposition 2 Let $n$ denote the neighbourhood size of each node in a network. The maximal Shannon entropy of an additive CA pattern resulting from the maxmin- $\omega$ system on this network increases with $\omega$ as $\log \omega+\log n$.

Proof We assume that $|\Sigma|>n$ to ensure that a node receives different states with a large probability. Then, for $m=\lceil\omega n\rceil$, we apply (8) to find that the maximal Shannon entropy is proportional to $\log m$ since the node is likely to receive $m$ different states. Expanding this gives the result.

So, given $\omega_{1}<\omega_{2}$, we know that the maximal Shannon entropy $\bar{S}^{(2)}$ of a maxmin- $\omega_{2}$ system will be greater than the maximal Shannon entropy $\bar{S}^{(1)}$ of a maxmin- $\omega_{1}$ system. However, this does not guarantee that the Shannon entropy $S^{(2)}$ of any maxmin- $\omega_{2}$ system will be greater than the Shannon entropy $S^{(1)}$, since $S^{(2)}$ might not necessarily be maximal. In fact, consider Fig. 6, which indicates where randomised Shannon entropies are likely to lie for each $m$; the logarithmic growth of the maximal entropy is echoed by the box plot. That is, for larger $\omega_{1}$ and $\omega_{2}$, if we select $S^{(1)}$ and $S^{(2)}$ uniformly at random (via their probabilities), then $S^{(2)}$ is likely to be larger than $S^{(1)}$ by a smaller amount. Additionally, note the tightening of the box plot as $m$ increases; this suggests that such an $S^{(2)}$ will not deviate considerably from $S^{(1)}$, especially if $\omega_{2}-\omega_{1}$ is small.

We move onto the analysis of word entropy as a function of $\omega$. For $\omega$ small, the reduced network will have small neighbourhood size. Using the same arguments as earlier, the fastest CA states will prevail, giving few state possibilities. This is equivalent to having a small alphabet such that a range of word lengths are likely to be observed (see inset, right, of Fig. 3 where, for small alphabet, a range of word lengths have large, non-zero probabilities of being observed). For a larger $\omega$, the reduced network will have a larger neighbourhood size such that more CA states are prevalent; the likelihood of observing $l$-words is therefore decreasing with $l$ (see inset, left, of Fig. 3). Thus, we expect word entropy to decrease with $\omega$.

Compared to the Shannon entropy, the exact trend of how $W$ varies with $\omega$ is tricky to calculate, suffice it to say that we have presented here heuristic arguments for developing such functions; these will be compared with the experimental results presented in the next section. 
Fig. 6 Shannon entropies generated uniformly at random. The entropy is calculated using (5) by selecting probabilities $p\left(s_{j}\right)$ uniformly at random, and letting $|\Sigma|=m$ such that the $x$ axis denotes the number of terms $m$ in the sum. For each $m$, we plot the distribution of $10^{5}$ such Shannon entropies

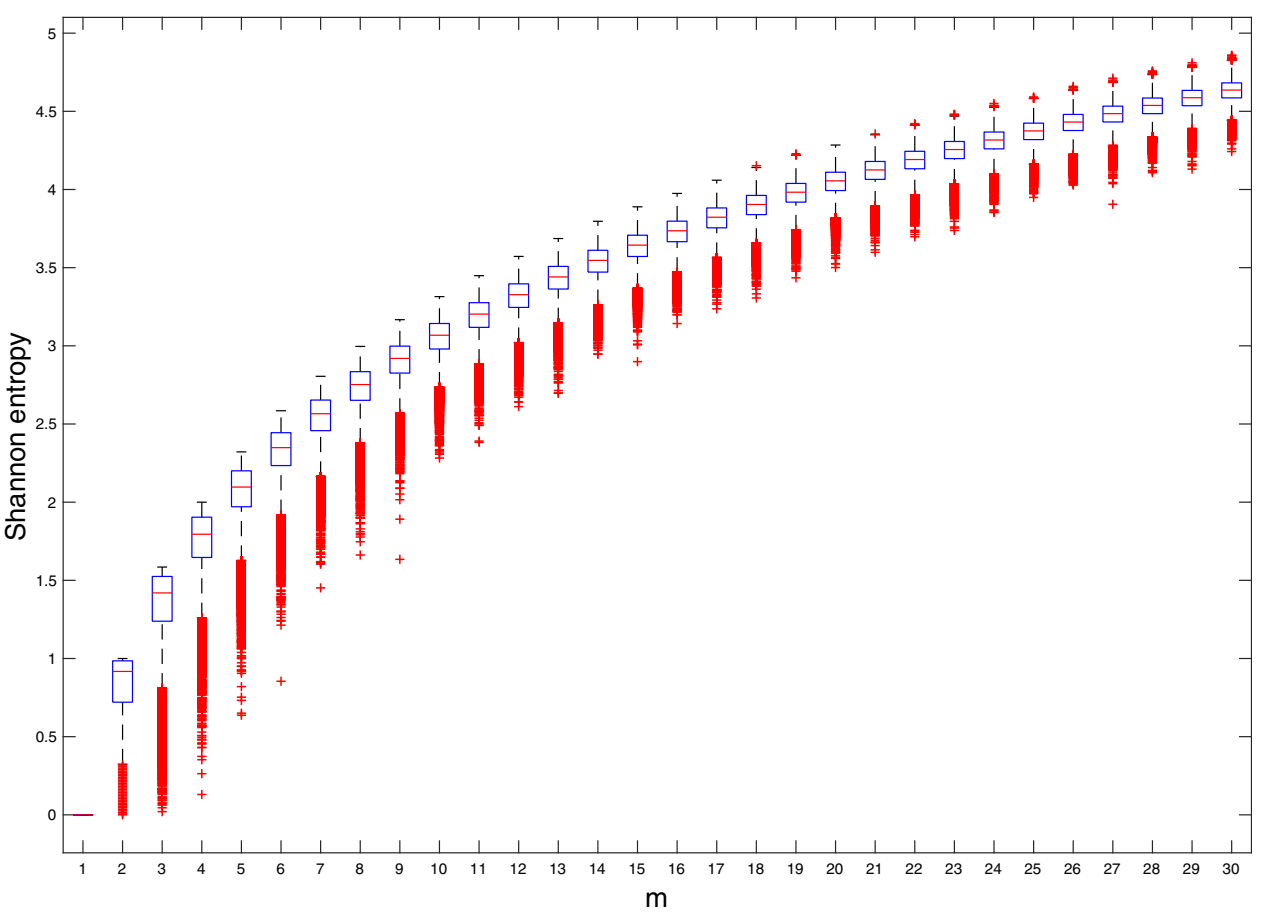

\section{Experimental results}

We now run the maxmin- $\omega$ system and implement the additive cellular automaton rule (3). The underlying network is regular, equivalent to the one-dimensional CA lattice, and we take network size $N=11$, where cells $N$ and 1 are connected. We record the asymptotic values of Shannon and word entropies, along with the asymptotic quantities that summarise the update times of the maxmin$\omega$ system itself. For this purpose, we require the following definitions.

Define the function $\mathcal{M}$ as the mapping $\mathcal{M}: \mathbb{R}^{N} \rightarrow \mathbb{R}^{N}$ whose components $\mathcal{M}_{i}$ are of the form of Eq. (1). We represent a system of $N$ such equations by the following.

$\mathbf{x}(k+1)=\mathcal{M}(\mathbf{x}(k))$

for $k \geq 0$, where $\mathbf{x}(k)=\left(x_{1}(k), x_{2}(k), \ldots, x_{N}(k)\right)$. Denote by $\mathcal{M}^{p}(\mathbf{x})$ the action of applying $\mathcal{M}$ to a vector $\mathbf{x} \in \mathbb{R}^{N}$ a total of $p$ times, i.e., $\mathcal{M}^{p}(\mathbf{x})=\underbrace{\mathcal{M}(\mathcal{M}(\cdots(\mathcal{M}}_{p \text { times }}(\mathbf{x})) \cdots))$.

Definition 4.1 If it exists, the cycletime vector of $\mathcal{M}$ is $\chi(\mathcal{M})$ and is defined as $\lim _{k \rightarrow \infty}\left(\mathcal{M}^{k}(\mathbf{x}) / k\right)$.

Definition 4.2 For some $k \geq 0$, consider the set of vectors $\mathbf{x}(k), \mathbf{x}(k+1), \mathbf{x}(k+2), \ldots \in \mathbb{R}^{N}$

where $\mathbf{x}(n)=\mathcal{M}^{n}(\mathbf{x}(0))$ for all $n \geq 0$. The set $x_{i}(k), x_{i}(k+$ $1), x_{i}(k+2), \ldots$ is called a periodic regime of $i \in \mathbb{N}$ if there exists $\mu_{i} \in \mathbb{R}$ and a finite number $\rho_{i}, \in \mathbb{N}$ such that $x_{i}\left(k+\rho_{i}\right)=\mu_{i}+x_{i}(k)$.

The period of the regime is $\rho_{i}$ and $\chi_{i}=\mu_{i} / \rho_{i}$ is the $c y$ cletime of $i$. The smallest $k$ for which the periodic regime exists is called the transient time.

Under our initial conditions, $K_{i}$ will be finite [see Heidergott et al. (2006), Theorem 12.7] and so, maxmin- $\omega$ always yields a periodic regime with the following systemwide quantities.

$$
K=\max _{i}\left\{K_{i}\right\}, \quad \rho=\operatorname{LCM}_{i}\left(\rho_{i}\right), \quad \chi=(1 / N) \sum_{i=1}^{N} \chi_{i} .
$$

Our experiments may best be described by the following.

\section{Algorithm 4.1}

1. Choose $\xi_{i}, \tau_{i} \in \mathbb{Z}$ both from the uniform distribution (with equal probability) taking largest value 5.

2. Choose an initial timing vector, $\mathbf{x}(0)=(0, \ldots, 0)$, and an initial $C A$ state $\mathbf{s}(0)$ uniformly (with equal probability) from the alphabet $\Sigma=\{0,1, \ldots, Z-1\}$.

3. Iterate the maxmin- $\omega$ system 100 times for each $\omega$ value from 0.05 to 1 , in steps of 0.05 (so there are 20 maxmin- $\omega$ systems to run).

4. For each maxmin- $\omega$ system, record the period $\rho$ and cycletime $\chi$, as well as the Shannon and word entropies.

5. Repeat above three steps 50 times to obtain, for each maxmin- $\omega$ system above, 50 independent transient times, periods, and cycletimes, as well as Shannon and word entropies. 

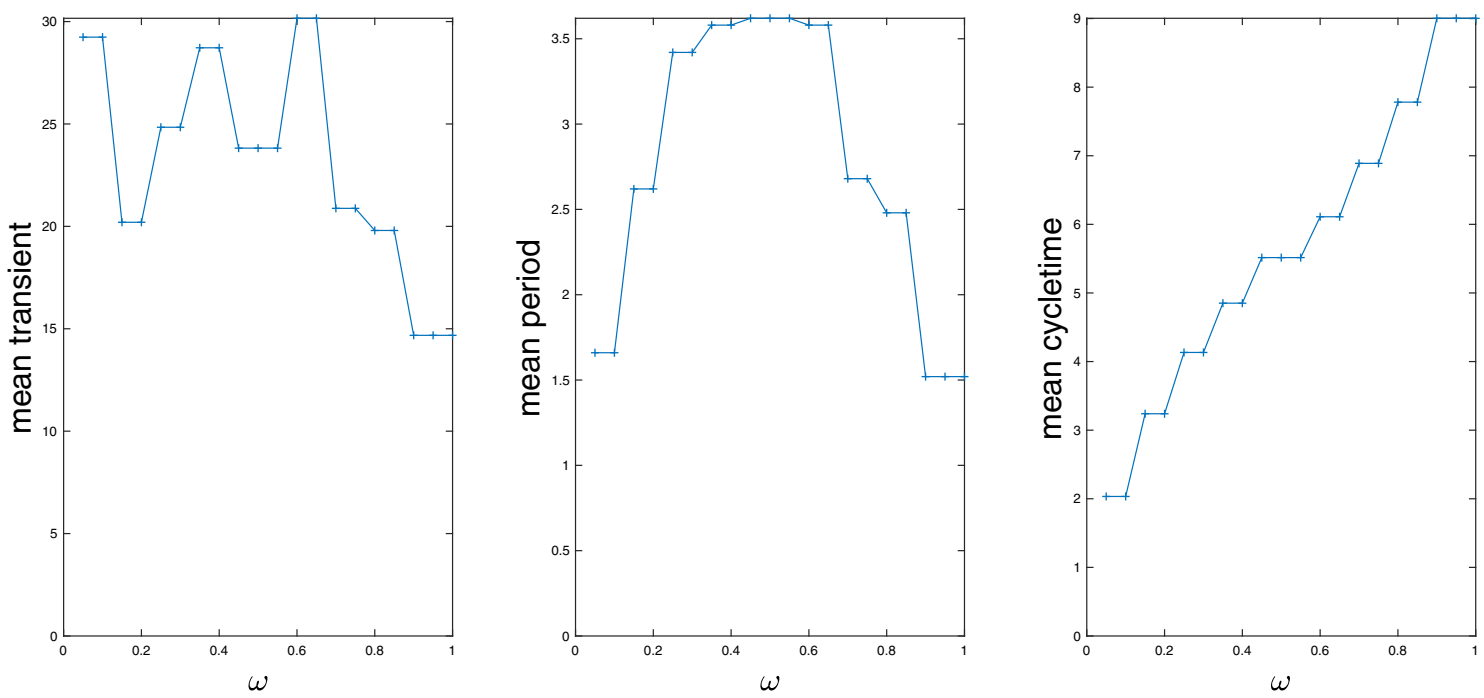

Fig. 7 Mean transient time, period and cycletime as a function of $\omega$ for a regular network of size 11 with neighbourhood size $n=9$. Each curve represents each of the alphabet sizes 2 to 10 (although these sizes are relevant only for the cellular automaton evolution)

6. For each maxmin- $\omega$ system, record the mean of the 50 transient times, periods, cycletimes, Shannon and word entropies obtained.

We vary the neighbourhood radius such that neighbourhood sizes explored are $n=3,5,7,9$, and 11. Thus, this algorithm is implemented on five regular networks of

(a)

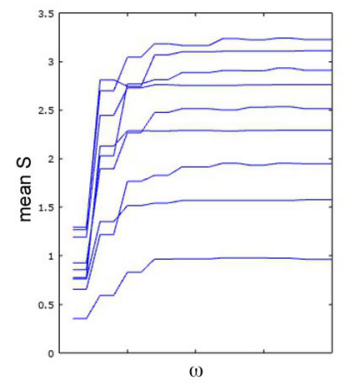

$n=9$

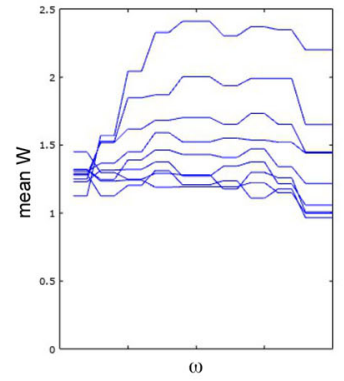

$n=11$
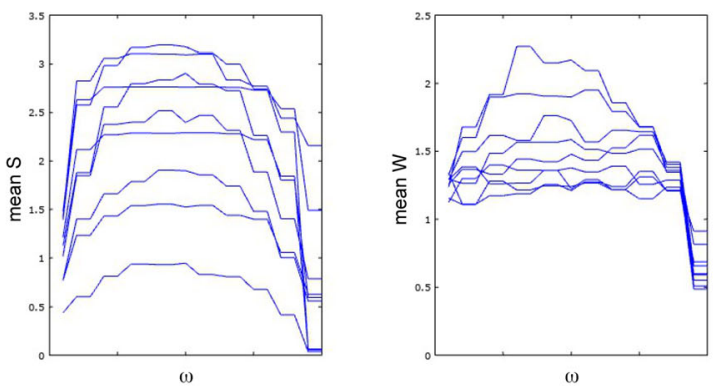

Fig. 8 (Colour online) Shannon and word entropy $S$ and $W$ for a regular size 11 network with neighbourhood sizes $n=9$, and 11 (results for $n=3,5$, and 7 similar to $n=9$ ). (a) Mean $S$ and mean $W$ versus $\omega$; each curve represents each of the alphabet size $Z=2$ to 10 , increasing connectivity. We also vary the alphabet size such that, for each of these networks, the algorithm is run for alphabet sizes $|\Sigma|=2,3, \ldots, 10$. Figures 7 and 8 summarise the results.

We first comment on the asymptotic values of the maxmin- $\omega$ update times. The 'bell' curve for both period and transient time was conjectured in Patel (2012), and is

(b)

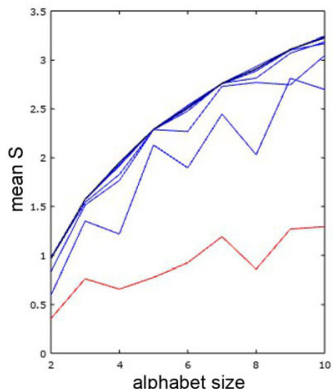

$n=9$

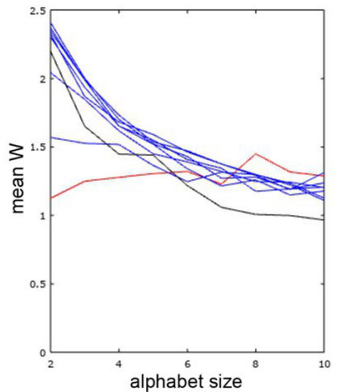

$n=11$
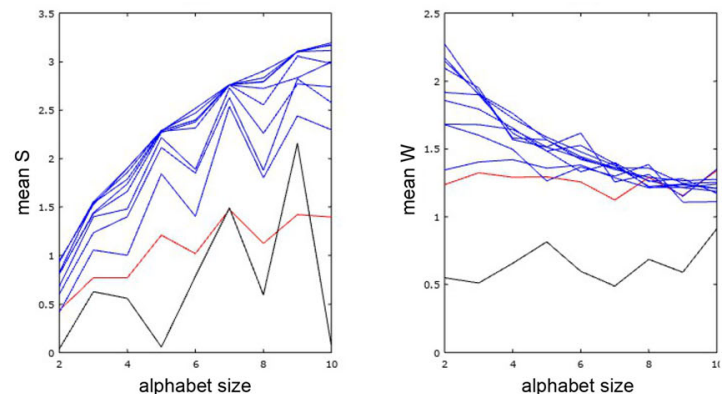

where a lower $Z$ corresponds to a lower curve. (b) Mean $S$ and mean $W$ versus $Z$; each curve represents each $\omega \in\{0.05,0.1, \ldots, 1\}$. Black: $\omega=1$, blue: $0.1 \leq \omega \leq 0.95$, red: $\omega=0.05$ 
evident for the period in Fig. 7. There is no evidence, however, to link the same shape to the Shannon or word entropy as functions of $\omega$, particularly when larger alphabet sizes are considered.

For all neighbourhood sizes except for $n=11$, the Shannon entropy is an increasing value with $\omega$ (see Fig. 8a), in agreement with our analytical predictions in the previous section. The word entropy behaves differently, however; it is not strictly decreasing with $\omega$. In fact, it is increasing for most alphabet sizes, with a sharp decrease at $\omega=1$; this decrease does fit with our heuristic arguments, but there is no evidence to suggest maximal $W$ at the smallest $\omega$ value (0.05). On the other hand, Fig. 8b agrees with our heuristic predictions when alphabet size is approximately greater than or equal to 8 ; that is, $W$ is a decreasing function of $\omega$ for a large alphabet size (the red curve is higher than the blue curve, which is higher than the black curve).

The logarithmic trend (8) of $S$ with alphabet size is most apparent-this is attained when all CA states are equiprobable, and it is maximal, supported by the black Shannon entropy curve in Fig. 8b, which is the $\omega=1$ case. As expected, the word entropy is decreasing with alphabet size, although the case $\omega=0.05$ is almost constant (see red line in Fig. 8b).

The exception seems to be the case $n=11$. This is where the network is fully connected; all nodes are neighbours of every other node. Here, the Shannon entropy does not follow a logarithmically increasing trend with $\omega$, instead following the bell-like curve referenced above, taking maximal value at approximately $\omega=0.5$. This Shannon entropy is minimal when $\omega=1$ (see black curve in Fig. 8b).

We end this section by combining the Shannon and word entropy results into one $(S, W)$-plane. Thus, for each $n$, consider a fixed alphabet size. This gives a pair (mean $S$, mean $W$ ) for each $\omega$ value. To find which of these produces the 'most complex' point, we take the following simple distance from the origin, for $\omega \in(0,1]$.

$d_{\omega}=\sqrt{(\text { mean } S)^{2}+(\text { mean } W)^{2}}$

Then, we are interested in $\omega^{*}=\arg \max _{\omega} d_{\omega}$, i.e., the $\omega$ values that maximise $d_{\omega}$. Often there exist more than one such $\omega$. For example, for $n=3$, $\omega^{*}=\{0.75,0.8,0.85,0.9,1\}$ for all alphabet sizes; in such cases, we take the mean of this list of $\omega^{*}$ values.

Thus, for each alphabet size, we have one $\omega^{*}$ value. Performing this calculation for all alphabet sizes up to 10 , we produce histograms indicating where such $\omega^{*}$ values congregate; these are shown in Fig. 9. A similar calculation finds, for a fixed $\omega$ value, the alphabet size that produces the 'most complex' $(S, W)$ point; we denote this alphabet size $Z^{*}$, also depicted in Fig. 9.

\section{Discussion}

We have demonstrated the effect of maxmin- $\omega$ dynamics on an additive cellular automaton. Thus, we have departed from synchronous elementary CA studies in two orthogonal ways: asynchrony was imposed via maxmin- $\omega$ and, secondly, the alphabet of state possibilities was extended. Overall, we have exhibited the properties of a deterministic asynchronous dynamical system that better reflects the internal decision-making processes of CA and its applications, such as epidemic spreading.

A synchronous CA is one whose contours would be horizontal in space-time; all cells update at the same real time. Moreover, such CA cell states are updated upon knowledge of all neighbourhood cell states. This implies that a synchronous $\mathrm{CA}$ is equivalent to the maxmin-1 system where all transmission times $\tau_{i j}$ and processing times $\xi_{i}$ are equal and constant. In fact, for $\omega=1$, we have previously shown that, even when $\tau_{i j}$ and $\xi_{i}$ are not all equal, the entropy measures $S$ and $W$ do not deviate significantly from the fully synchronous case (Patel 2012). This is primarily due to the bijection between states on maxmin- 1 contours and states on horizontal contours of a synchronous CA; all neighbourhood information is retained in both systems (Patel and Broomhead 2014). As $\omega$ is decreased from 1 , more of this neighbourhood information is lost such that, when $\omega \approx 0$, the system is losing the most information; prediction of CA behaviour is thus more difficult with decreasing $\omega$.

Indeed, whilst we previously noted some correspondence in complexity between timing and CA pattern when the alphabet $\Sigma$ was binary (Patel 2012, 2016), here, we have shown that such a link is simplistic; an extended alphabet generates additional facets to the story of complexity. Notwithstanding this, we have been able to analytically predict the tendency for complexity by thinking about the asymptotic behaviour of maxmin- $\omega$ - the socalled reduced system, which is effective for larger alphabet sizes (at least larger than the neighbourhood size). Thus, we claim that the essence of the maxmin- $\omega$ system is best captured by a CA with a larger number of states than two; here, complexity doesn't follow simple bell-like curves, unless the network is fully connected, such as the $n=11$ case. Interestingly, however, we note that the $(S, W)$-plane offers some support to the argument that the most complex patterns occur when $\omega \approx 0.5$ (see Fig. 9).

To conclude, we have introduced a larger alphabet to a maxmin- $\omega$ update scheme for cellular automata, opening 
Fig. 9 Frequency of $\omega^{*}$ and $Z^{*}$ values that yield the most complex CA patterns. Left: Frequency of $\omega^{*}$ values. Right: Frequency of $Z^{*}$ values. We present results for neighbourhood sizes $n=9$ and $n=11$ as they show the most variation
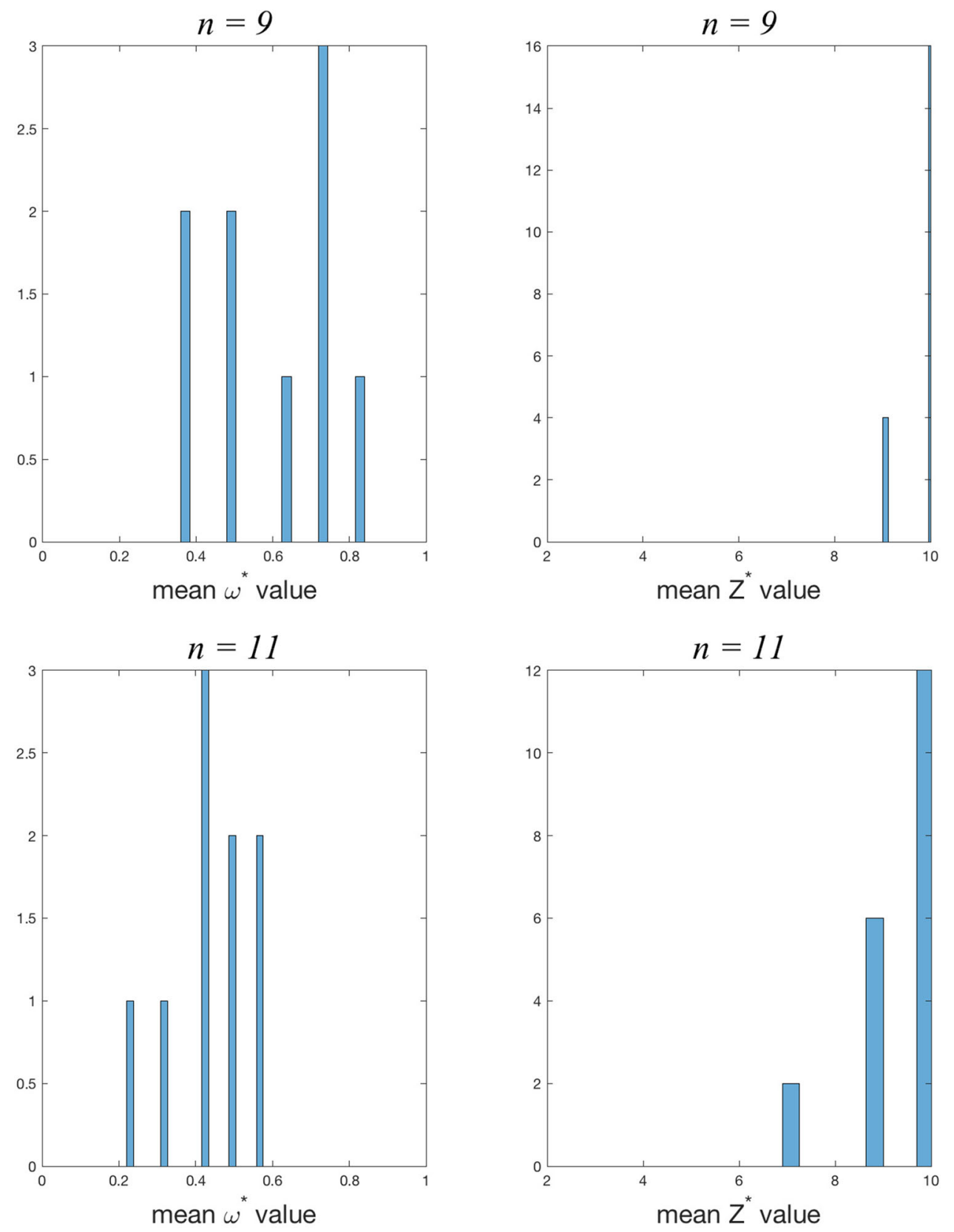

up many avenues for future work. For instance, the reduced system is smaller, and more predictable (Sect. 3) such that a strict sequence of updated nodes can be extracted. Thus, we can assign a probability of update to each cell; we would expect this probability to depend on $\tau_{i j}$ and $\xi_{i}$. This suggests that maxmin- $\omega$ can yet be described in terms of traditional, probabilistic, asynchronous models; indeed, we have previously proposed maxmin-1 as a system that delves into how updating probabilities might be generated by local 'handshaking' of nodal states (Patel and Broomhead 2014). It would be interesting to see how maxmin- $\omega$ fits into the more generalised class of probabilistic asynchronous CA, such as $m$-asynchronous CA (Dennunzio et al. 2013b).

Finally, we are currently thinking about the maxmin- $\omega$ system on various topologies; this would allow a better reflection of applications such as information spreading over social networks. Along these lines, Marr et al. have looked at the impact of metabolic network structures and scale-free networks on the resulting complexity of binary CA dynamics (Marr et al. 2007), finding that metabolic networks 'dampen' the effect of perturbations in the network structure; complexity is thus reduced in such 'nonregular' graphs. The analytical work of this paper (such as 
Proposition 2) suggests that the general properties of the Shannon and word entropies are independent of the global network structure, beyond local neighbourhood information; this conjecture itself gives a tantalising suggestion of complexity under maxmin- $\omega$ being universal. It would therefore be interesting to test this by comparing our methods with that of Marr et al. (2007) and to implement maxmin- $\omega$ CA on arbitrary network structures. Already, the case $n=11$ is in contrast to the hypothesis of Marr et al. (2007), but we reiterate that the predictable reduction to a smaller system, which would be independent of $\omega$, can aid this study significantly. That is, prediction of the behaviour of a threshold-like state system is straightforward with a maxmin- $\omega$ model.

Acknowledgements We wish to thank the anonymous reviewers for their detailed feedback and suggestions for improving this work.

Open Access This article is licensed under a Creative Commons Attribution 4.0 International License, which permits use, sharing, adaptation, distribution and reproduction in any medium or format, as long as you give appropriate credit to the original author(s) and the source, provide a link to the Creative Commons licence, and indicate if changes were made. The images or other third party material in this article are included in the article's Creative Commons licence, unless indicated otherwise in a credit line to the material. If material is not included in the article's Creative Commons licence and your intended use is not permitted by statutory regulation or exceeds the permitted use, you will need to obtain permission directly from the copyright holder. To view a copy of this licence, visit http://creativecommons. org/licenses/by/4.0/.

\section{Appendix: Proof of Proposition 1}

When all CA states are equiprobable, we have that $S=$ $\log Z$ from (8), where $Z=|\Sigma|$, the number of CA states. The Shannon entropy can be rewritten as

$S=\sum_{j=1}^{|\Sigma|} p\left(s_{j}\right) \log _{2}\left(1 / p\left(s_{j}\right)\right)$.

Now we use the fact that the logarithm function is concave and apply Jensen's inequality, as follows (Jensen 1906).

$$
\log \left(\frac{\sum_{j=1}^{Z} p\left(s_{j}\right) \cdot 1 / p\left(s_{j}\right)}{\sum_{j=1}^{Z} p\left(s_{j}\right)}\right) \geq \frac{\sum_{j=1}^{Z} p\left(s_{j}\right) \cdot \log \left(1 / p\left(s_{j}\right)\right)}{\sum_{j=1}^{Z} p\left(s_{j}\right)}
$$

$$
\begin{aligned}
& \log \left(\sum_{j=1}^{Z} p\left(s_{j}\right) \cdot 1 / p\left(s_{j}\right)\right) \geq \sum_{j=1}^{Z} p\left(s_{j}\right) \cdot \log \left(1 / p\left(s_{j}\right)\right) \\
& \text { since } \sum_{j=1}^{Z} p\left(s_{j}\right)=1 .
\end{aligned}
$$

$\log Z \geq S$

\section{References}

Aso H, Honda N (1985) Dynamical characteristics of linear cellular automata. J Comput Syst Sci 30(3):291-317

Backlund V-P, Saramäki J, Pan RK (2014) Effects of temporal correlations on cascades: threshold models on temporal networks. Phys Rev E 89(6):062815

Bouré O, Fates N, Chevrier V (2012) Probing robustness of cellular automata through variations of asynchronous updating. Nat Comput 11(4):553-564

Cattaneo G, Formenti E, Manzini G, Margara L (2000) Ergodicity, transitivity, and regularity for linear cellular automata over $\mathrm{zm}$. Theor Comput Sci 233(1-2):147-164

Cattaneo G, Dennunzio A, Margara L (2002) Chaotic subshifts and related languages applications to one-dimensional cellular automata. Fund Inform 52(1-3):39-80

Cattaneo G, Dennunzio A, Margara L (2004) Solution of some conjectures about topological properties of linear cellular automata. Theor Comput Sci 325(2):249-271

Chaudhuri PP, Chowdhury DR, Nandi S, Chattopadhyay S (1997) Additive cellular automata: theory and applications, vol 1 . Wiley, New York

Dennunzio A, Di Lena P, Formenti E, Margara L (2013a) Periodic orbits and dynamical complexity in cellular automata. Fund Inform 126(2-3):183-199

Dennunzio A, Formenti E, Manzoni L, Mauri G (2013b) masynchronous cellular automata: from fairness to quasi-fairness. Nat Comput 12(4):561-572

Dennunzio A, Formenti E, Manzoni L, Mauri G, Porreca AE (2017) Computational complexity of finite asynchronous cellular automata. Theor Comput Sci 664:131-143

Dennunzio A, Formenti E, Manzoni L, Margara L, Porreca AE (2019) On the dynamical behaviour of linear higher-order cellular automata and its decidability. Inf Sci 486:73-87

Fatès NA, Morvan M (2005) An experimental study of robustness to asynchronism for elementary cellular automata. Complex Syst 16:1-27

Gevertz JL, Torquato S (2006) Modeling the effects of vasculature evolution on early brain tumor growth. $\mathrm{J}$ Theor Biol 243(4):517-531

Granovetter M (1978) Threshold models of collective behavior. Am J Sociol 83(6):1420-1443

Guan P-H, He Y (1986) Exact results for deterministic cellular automata with additive rules. J Stat Phys 43(3-4):463-478

Gutowitz H, Langton C (1995) Mean field theory of the edge of chaos. In: European conference on artificial life. Springer, pp 52-64

Heidergott B, Olsder GJ, Van der Woude J (2006) Max Plus at work: modeling and analysis of synchronized systems: a course on 
Max-Plus algebra and its applications. Princeton University Press, London

Jensen JLWV (1906) Sur les fonctions convexes et les inégalités entre les valeurs moyennes. Acta Math 30(1):175-193

Lopez-Ruiz R, Mancini HL, Calbet X (1995) A statistical measure of complexity. Phys Lett A 209(5-6):321-326

Manzini G, Margara L (1999) A complete and efficiently computable topological classification of d-dimensional linear cellular automata over zm. Theor Comput Sci 221(1-2):157-177

Marr C, Hütt M-T (2005) Topology regulates pattern formation capacity of binary cellular automata on graphs. Phys A Stat Mech Appl 354:641-662

Marr C, Müller-Linow M, Hütt M-T (2007) Regularizing capacity of metabolic networks. Phys Rev E 75(4):041917

McCulloch WS, Pitts W (1943) A logical calculus of the ideas immanent in nervous activity. Bull Math Biophys 5(4):115-133

Nagel K, Schreckenberg M (1992) A cellular automaton model for freeway traffic. Journal de physique I 2(12):2221-2229

Patel E (2012) Maxmin-plus models of asynchronous computation, $\mathrm{Ph} . \mathrm{D}$. thesis, University of Manchester

Patel EL (2016) Maxmin- $\omega$ : a simple deterministic asynchronous cellular automaton scheme. In: El Yacoubi S, Wąs J, Bandini S (eds) Cellular automata. ACRI 2016. Lecture notes in computer science, vol 9863. Springer, Cham, pp 192-198

Patel E, Broomhead D (2014) A max-plus model of asynchronous cellular automata. Complex Syst 23(4):313-341
Piotrowska MJ, Angus SD (2009) A quantitative cellular automaton model of in vitro multicellular spheroid tumour growth. J Theor Biol 258(2):165-178

Rényi A (1961) On measures of entropy and information. In: Proceedings of the fourth Berkeley symposium on mathematical statistics and probability, Volume 1: contributions to the theory of statistics, the regents of the University of California

Schönfisch B, de Roos A (1999) Synchronous and asynchronous updating in cellular automata. BioSystems 51(3):123-143

Thul R, Coombes S, Laing CR (2016) Neural field models with threshold noise. J Math Neurosci 6(1):3

Watts DJ (2002) A simple model of global cascades on random networks. Proc Natl Acad Sci 99(9):5766-5771

White SH, Del Rey AM, Sánchez GR (2007) Modeling epidemics using cellular automata. Appl Math Comput 186(1):193-202

Wilson HR, Cowan JD (1972) Excitatory and inhibitory interactions in localized populations of model neurons. Biophys J 12(1):1-24

Wolfram S (1983) Statistical mechanics of cellular automata. Rev Mod Phys 55:601-644. https://doi.org/10.1103/RevModPhys.55. 601

Publisher's Note Springer Nature remains neutral with regard to jurisdictional claims in published maps and institutional affiliations. 